\title{
Meeting of the Eastern Mediterranean Regional Technical Advisory Group (RTAG) on immunization ${ }^{1}$
}

Citation: Meeting of the Eastern Mediterranean Regional Technical Advisory Group (RTAG) on immunization. East Mediterr Health J. 2020;26(9):11411142 https://doi.org/10.26719/2020.26.9.1141

Copyright (C) World Health Organization (WHO) 2020. Open Access. Some rights reserved. This work is available under the CC BY-NC-SA 3.0 IGO license (https://creativecommons.org/licenses/by-nc-sa/3.o/igo).

\section{Introduction}

The COVID-19 pandemic has created unprecedented challenges for immunization programmes in the Eastern Mediterranean Region. In order to assess the impact of COVID-19 and scrutinize the current status of immunization goals in the Region, the World Health Organization Regional Office for the Eastern Mediterranean (WHO/ EMRO), Cairo, Egypt, held a meeting of the Regional Technical Advisory Group (RTAG) on immunization during 13-14 April 2020 (1). The meeting was conducted virtually (due to the COVID-19 pandemic situation).

The objectives of the meeting were to:

- review regional progress, challenges and constraints facing the achievement of the goals of the Eastern Mediterranean Vaccine Action Plan (EMVAP) and provide advice on the way forward;

- brief RTAG members on the progress in verification of elimination of measles and rubella and hepatitis B control in the Region;

- review the standard operating procedures (SOPs) of the RTAG; and

- discuss the impact of the COVID-19 pandemic on immunization programmes in the Region and measures to mitigate it.

\section{Summary of discussions}

The current COVID-19 pandemic may result in a substantial negative impact on immunization programmes in the Region. Initial information from many countries indicates postponement of vaccination campaigns and underutilization of routine immunization services. An accumulation of susceptible populations may have serious consequences for vaccine-preventable diseases (VPD) morbidity and mortality. Regional routine vaccination coverage has increased in recent years despite the challenges facing the Region. However, around 3 million infants - almost 1 in 5 - still miss receiving their basic vaccines (2).

Commendable progress has taken place towards measles and rubella elimination in the Region, with half of its countries having either achieved elimination or progressed well towards it. However, the occurrence of measles outbreaks in several countries and the failure of five countries to achieve maternal and neonatal tetanus (MNT) elimination are persistent problems.

Several outbreaks of VPDs have occurred recently in the Region, mainly affecting countries with low routine coverage. Improving immunization coverage, introducing booster doses and improving regional and national capacity for early detection and rapid response are required.

While commendable efforts to improve immunization data quality are ongoing in several countries, the current overall situation of immunization data quality hampers optimal decision-making. However, remarkable progress has taken place in establishing processes for verification of elimination and control goals at the regional level.

\section{Recommendations}

\section{To WHO}

- Promoting the use of absolute numbers of unvaccinated children along with vaccination coverage to monitor programme performance, especially in countries with high numbers of unvaccinated children.

- Support countries of the Region in submitting high quality applications to GAVI to introduce second doses of measles and rubella vaccines.

- Responding to the demand of populations and decision-makers for a vaccine against COVID-19, as an opportunity to promote the unique importance of vaccination, as most cost-effective tool to prevent and control disease outbreaks, epidemics and pandemics.

- Finalizing, printing and disseminating the regional guide for verification of the hepatitis $B$ reduction target and conducting briefing sessions for countries on the verification process, as soon as possible.

- Ensuring continued support to the Expanded Programme on Immunization (EPI) to streamline the sources and flow of immunization data in order to ensure the coherence and standardization of immunization data reporting.

\section{To Member States}

- Ensuring that countries of the Region document the impact of the COVID-19 pandemic on national immunization programmes and monitor vaccine availability.

\footnotetext{
This report is based on the Summary Report on the Meeting of the Eastern Mediterranean Regional Technical Advisory Group (RTAG) on immunization, 13-14 April 2020, virtual meeting.
} 
- Monitoring susceptibility to measles and closing the immunity gap among all population groups, including refugees, immigrants, health-care workers and other high-risk groups.

- Ensuring that countries make plans for catch-up vaccination of missed children, using appropriate strategies, as soon as possible, when the COVID-19 pandemic situation allows.

- Involving the national immunization technical advisory group (NITAG), relevant partners and stakeholders in decision-making on the implementation of immunization activities during the COVID-19 pandemic.

- Designing appropriate strategies for the integrated delivery of essential services during the COVID-19 pandemic where possible, and in the post-pandemic period.

- Ensuring that countries of the Region maintain high-quality VPD surveillance and alert functions to safeguard the early detection of VPD outbreaks and timely response to them.

- Encouraging countries of the Region to regularly share their subnational immunization data to ensure identification of missed children and achieve national and regional immunization goals.

\section{References}

1. World Health Organization Regional Office for the Eastern Mediterranean (WHO/EMRO). Summary Report on the Meeting of the Eastern Mediterranean Regional Technical Advisory Group (RTAG) on immunization. Cairo: WHO/EMRO; 2020.

2. World Health Organization Regional Office for the Eastern Mediterranean (WHO/EMRO). WHO urges countries to continue lifesaving immunization services during the COVID-19 pandemic. Cairo: WHO/EMRO; 2020 (http://www.emro.who.int/media/ news/who-urges-countries-to-continue-lifesaving-immunization-services-during-the-covid-19-pandemic.html). 